\title{
Engaging Pedestrians in Designing Interactions with Autonomous Vehicles
}

\section{Himanshu Verma}

himanshu.verma@unifr.ch

University of Fribourg \& University of Applied

Sciences of Western Switzerland

Switzerland

\section{Guillaume Pythoud}

guillaume.pythoud@unifr.ch

University of Fribourg

Fribourg, Switzerland

\section{Denis Lalanne}

denis.lalanne@unifr.ch

University of Fribourg

Fribourg, Switzerland

\section{Florian Evéquoz}

florian.evequoz@unifr.ch

University of Fribourg \& University of Applied

Sciences of Western Switzerland

Switzerland

\section{Grace Eden}

G.Eden@mmu.ac.uk

Manchester Metropolitan University

Manchester, UK

\section{ABSTRACT}

Driverless Passenger Shuttles are operating as a public transport alternative in the town of Sion, Switzerland since June' 16 , and traversing the populated commercial and residential zones of the city center. The absence of a human driver and the lack of dedicated AV-pedestrian interface makes it challenging for road users (pedestrians, cyclists, etc.) to understand the intent or operational state of

Permission to make digital or hard copies of part or all of this work for personal or classroom use is granted without fee provided that copies are not made or distributed for profit or commercial advantage and that copies bear this notice and the full citation on the first page. Copyrights for third-party components of this work must be honored. For all other uses, contact the owner/author(s).

CHI'19 Extended Abstracts, May 4-9, 2019, Glasgow, Scotland UK

() 2019 Copyright held by the owner/author(s).

ACM ISBN 978-1-4503-5971-9/19/05.

https://doi.org/10.1145/3290607.3312864 


\section{KEYWORDS}

Autonomous Vehicles (AVs); Communicating AV Intentions; AV-Pedestrian Interaction; Public Transport; Urban Mobility; Co-design. the vehicle and negotiate road usage. In this article, we present a co-design study aimed at informing the design of interactive communication means between pedestrians and autonomous vehicles (AVs). Conducted in two stages with the local community - which is accustomed to the AV's ecosystem and has interacted with it on a daily basis- the study highlights the interactive experiences of road users, and furnishes contextualized design guidelines to bridge the communication with the pedestrians.

\section{INTRODUCTION}

Forecasts concerning the Autonomous Vehicles (AVs) have promised profound transformations in the urban mobility. AVs may alter cities by making them highly scattered, increase connectivity with the rural and sub-urban areas, and change the nature of public transport [7, 13, 15]. Besides the projected overhaul of the very fabric of mobility, the interaction design challenges arising due to the nuanced and manifold interactions between humans and $\mathrm{AVs}$ have to be considered, and grounded in the everyday socio-cultural dynamics. $\mathrm{HCl}$ and Interaction Design researchers have examined and consolidated these interactions in two ways: $a$ ) the in-vehicle interaction with the driver and other passengers (for example, $[4,8,12]$ ), and $b$ ) the interactions with the other road users such as pedestrians, cyclists, other drivers, etc. (for example, $[2,9,10])$. The work presented in this article belongs to this latter class of interactions.

Non-verbal indicators such as gaze, gestures, and postures are used by traditional drivers to signal their awareness of other road users (at a crosswalk, for example) and as a sign of their attentiveness [9, 10]. These signs are demonstrated to induce the feeling of reassurance and confidence amongst the road users $[5,16]$. Owing to their risk-averse nature, the $A V s$ are considered by experts and manufacturers as a significant step towards a "pedestrian-centered urbanity" [1, 14]. Still, the absence of a human driver and the lack of means of embedded interactions (non-verbal signals from human operators, and their complementary replacements embedded within the vehicle) to communicate the intentions could in-turn affect the trust and overall social acceptance of AVs [9].

Higher level of perceived trust amongst the pedestrians for AVs was reported in the surveys conducted by Hulse et al. [6], which could be attributed to the promise of reduced human errors in the case of AVs. Furthermore, the lack of a human driver and unexpected behaviors on part of the AVs were observed to impede the development of trust and confidence amongst the road users [17]. Eden et al. [3] observed explicit communication signals directed towards the pedestrians from the stewards aboard a self-driving passenger shuttle, signifying the autonomous nature of the vehicle. Road users were also observed to react reassuringly and predictably when the AV's intent was communicated through technological means [11]. Therefore, to compensate for the negative effects arising due to the lack of human drivers, and the non-verbal signals to convey the drivers' intent, Lundgren et al. [9] and Mahadevan et al. [10] suggest the transfer of intent from the driver to the vehicle or the environment. This, in-turn entails comprehending the positive experiences and difficulties faced by 


\section{BACKGROUND \& CONTEXT}

In the town of Sion, Switzerland, autonomous passenger shuttles (Navya Arma - https://navya. tech/en/ - similar to mini-buses) are operating as a public transport alternative, and connecting the residential and commercial zones in the city center since June' 16 . The City Council in collaboration with a public transport service provider (PostBus), operate two shuttles everyday between 13:00 and 18:00. The situation is unique, as it is the first deployment of $\mathrm{AVs}$ on public roadways in Switzerland.

The trajectory of the shuttles intersects with the pedestrian-heavy zones several times during a single trip, suggesting a high level of interactivity between the shuttle and pedestrians. In addition, the local community is accustomed to the shuttle's operations and attributes as they have been interacting with the shuttle for 18 months before this research work began. Owing to this unique context and the lack of existing research on AV-Pedestrian Interactions that focuses on the wider spectrum of pedestrian behaviors and urban contexts (beyond roundabouts and crosswalks), the immediate transfer and applicability of design knowledge to our context is not straightforward. In addition, grounding the design of tools communicating AV's awareness of pedestrians and intent within the urban context can lead to a greater acceptability and trust in AVs [1]. Therefore, we conducted a co-design study with the local community to understand their interactive experiences with the shuttle and expectations about the use of $\mathrm{AVs}$ in public transport, and to subsequently inform the design of interactive communication tools. pedestrians who have interacted with the $\mathrm{AVs}$, and are accustomed to their behaviors and attributes. In our unique research context, we address this by conducting co-design sessions with residents of Sion (Switzerland) as described in 'Background \& Context'.

\section{CO-DESIGNING AV-PEDESTRIAN INTERFACES}

Two co-design sessions were organized with the voluntary residents of the city and a representative of the public transport service provider (PostBus). The subjects were recruited through advertisements in local forums. Separated by a period of two weeks, the purpose of these sessions was to instigate dialogue between the different stakeholders, and to elicit their opinions concerning $a$ ) residents' lack of awareness about the shuttle's operational states and how it influences their perception of it, $b$ ) the technological means and channels of representing these intentions and their positioning, and $c$ ) the importance of these representations in different sections of the shuttle's itinerary. Activities and discussions in both the sessions were audio-recorded, and later transcribed for analysis.

\section{Mapping Technological Affordances and Intent}

12 participants ( 4 females, 8 males) were split into 4 groups corresponding to their preferred means of commuting (pedestrians, cyclists, skateboarders, and car drivers). Each group was provided with 2 sets of cards (Figure 1) corresponding to 1) different operational states of vehicles (stopping, accelerating, slowing, obstacle detection, etc.), and 2) various technological modes of communicating information (sound, text, animated eyes/faces, etc.). In addition, an annotated map of the shuttle's itinerary including crosswalks, roundabouts, pedestrian zones, was provided to each group. Next, the groups were asked to discuss the implications resulting from the lack of driver, and prioritize 5 most important intentions of the vehicle that need to be communicated to pedestrians and the technological means of communicating them. Furthermore, the groups were also asked to annotate the sections of shuttle's itinerary where communicating intent and awareness would be of importance.

\section{Situating Interfaces}

The second session was designed to comprehend the pedestrians' preferences regarding the placement of the AV-Pedestrian interfaces (on the vehicle, on the urban infrastructure, etc.), informed by the pedestrians' perceptual experiences and interactions with the driverless shuttle. 14 participants were randomly assigned to 4 groups of 3-4 members, with half of the participants coming from the previous session.

Next, each group was supplied with $a$ ) a 3D printed miniaturized replica of the shuttle, $b$ ) a set of printed stickers (with standard set of icons including eyes \& mouth indicating different emotions and sense of urgency), and c) Five A4-sized sheets corresponding to the 5 intentions which were prioritized by the participants in the first session (start, accelerate, decelerate, brake, and awareness 


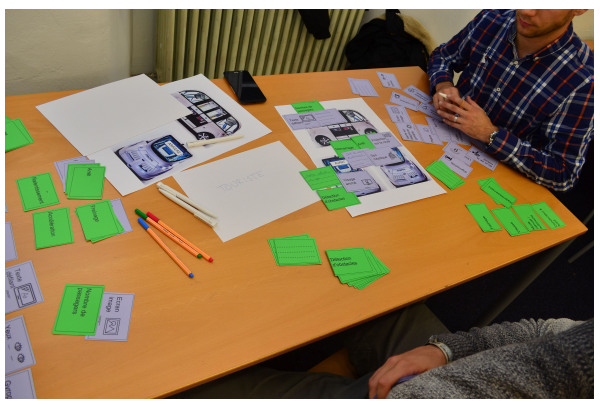

Figure 1: A snapshot from the first session.

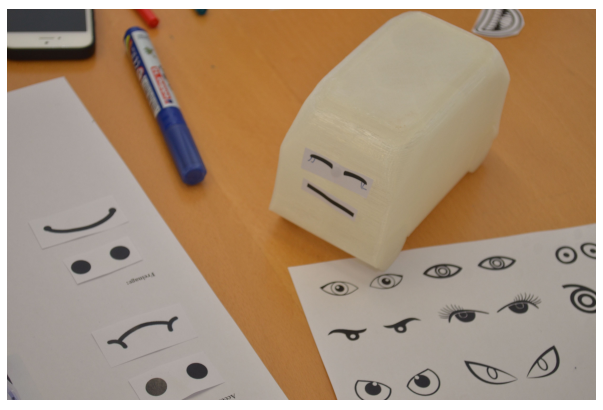

Figure 2: Participants trying out different animated faces in the second session.

\section{DISCUSSION QUOTES}

"You don't want them all to become into CocaCola trucks!"

"Humanizing the shuttle (by displaying animated eyes/faces) is a good way to interact with pedestrians who are new to AVs, however is it a practical and functional means of communication in the long term?" of pedestrians). The groups were then asked to either glue the combination of stickers, or sketch an interface directly upon the sheets, which could best signify and communicate the respective intention marked upon the sheet. The groups could try the combination of stickers upon the 3D printed replica, and could also indicate the surface(s) upon which such an interface can be localized (see Figure 2). Following this creative phase, the groups participated in an open discussion about ways in which road users can build trust and confidence in $\mathrm{AV}$ s.

\section{ANALYSIS AND RESULTS}

Acknowledging the high interactivity with the shuttle, especially in pedestrian priority zones (in the city-center) followed by crosswalks and round-abouts, the participants unanimously indicated their consensus about the different $\mathrm{AV}$ intentions that should be explicitly communicated to pedestrians. The analysis of the audio recordings revealed that participants reflected upon the attributes of the $A V$ 's intentions, and what constitutes these intentions. As a result, the current operational state of the shuttle and the subsequent action that the vehicle's $\mathrm{Al}$ is planning on making, was assigned as the intention of the vehicle -1) running ("Currently running and will stay running"), 2) slowing ("Currently running but will stop soon"), 3) halted ("Currently stopped and will stay stopped"), 4) starting ("Currently stopped but will start soon"), and 5) awareness of pedestrians and the depth of the shuttle's vision i.e. what and how far can the shuttle "see" The participants also highlighted the need for simplistic representations - preferential use of iconography and pictographs - that are understandable to a wide range of the population, and do not induce additional cognitive load amongst pedestrians. The use of text was discouraged owing to the bilingual demographics of the city, unsuitability for children, and their existing usage in public transportation to indicate destination and direction of operation.

In addition, a combination of recurrent multi-modal means (visual and auditory signs from the vehicle, as well as embedding ambient awareness in the urban landscape) were suggested as a way of rendering the awareness perceptible and readily accessible. However, the participants also warned of the dangers of information overload with the proliferation of $A V s$ on urban streets and different manufacturers using different set of symbols. One participant quoted the problem as "You don't want them all to become into Coca-Cola trucks!". Consequently, a desire for standardized universal set of symbols to communicate awareness and intent was expressed by the participants. The use of traditional traffic light colors in representing these signals was suggested as a way of ensuring clarity and straightforward interpretation by the pedestrians (for example, using green color to signal that the shuttle is stopped and pedestrians can cross the street). These findings are in line with previous works (for example, $[2,10]$ ), and subsequently extend the scope of research on AV-Pedestrian Interaction by engaging participants with prior experience of interacting with the $A V$ in naturalistic urban settings. 


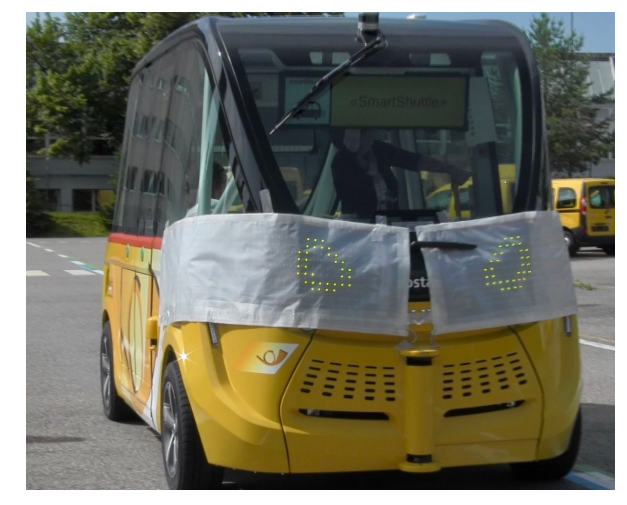

Figure 3: The Intent Communication Interface attached to the driverless shuttle, and displaying the Anthropomorphic signal corresponding to intention "running".
Furthermore, anthropomorphizing the intent information, for example, by the use of animated faces on the AV's front surface, was regarded as a transitory phase leading up to the time when a wider set of population is accustomed to the $\mathrm{AV}$ ecosystem, and have established confidence and trust in them. One participant questioned this aspect as "Humanizing the shuttle (by displaying animated eyes/faces) is a good way to interact with pedestrians who are new to AVs, however is it a practical and functional means of communication in the long term?".

Considering the manner of representing the aforementioned intentions, however, no agreement was reached, which also led to significant discussions during the open sessions. Based on their past experiences and expectations, the participants argued about the choice of one kind of representation over another, while elaborating the pros and cons of each kind. Participants also ascribed different priorities to the awareness of various $A V$ intentions owing to the movement of the vehicle. For example, communicating the intention of a shuttle which is moving or slowing down was given a higher precedence as compared to communicating the intention of a shuttle that is stationary. Moreover, effective and seamless communication of AV's intent was considered of utmost importance at crosswalks and pedestrian-priority zones rather than sidewalks and bus stops. Other road users (especially, cyclists and car drivers) who often come across the driverless shuttle from its rear side stated a need for visual signals which are rapidly perceivable, leverage peripheral vision, reduce cognitive load, and capable of guiding traffic.

\section{DISCUSSION AND FUTURE WORK}

The analysis of discussions, sketches, and annotations from the co-design study emphasized the lack of pedestrians' awareness about the AV's intentions and a unanimous desire for this awareness. In addition, we also observed a gap concerning the adequate manner of communicating this awareness. Moreover, this collaborative endeavor which engaged local residents and the representative of public transport service provider manifested as a framework for grounding the challenges faced by pedestrians while interacting with the driverless shuttle and their expectations. This effort convinced the public transport company of the need for immediate action regarding the communication of AVs' intentions to the pedestrians, and consequently paved the way for future exploration of visual signals which can foster the development of trust and social acceptance of autonomous public transportation. As a result, we ( $\mathrm{HCl}$ researchers and public transport company) decided to design an intent communication interface that can be attached to the vehicle, as other means illustrated by Mahadevan et al. [10] were not contextually possible either due to state regulations (signals in urban settings) or were perceived intrusive by residents (notification on mobile devices).

In our future work, focusing specifically on the visual means of communicating AVs' intentions to pedestrians, we are planning to compare four types of signals, namely textual instructions, symbolic patterns, metaphorical pictographs, and anthropomorphic animations. We have designed two flexible 


\section{ACKNOWLEDGEMENTS}

We would like to thank our colleagues from PostBus (Switzerland) including Anne-Claire Pliska, Lukas Krienbühl, Jörg Michel, and Flavien Claivaz who contributed to the study. The anonymous reviewers deserve special appreciation for their suggestions. Finally, our special thanks to the participants of our study.
LED panels (see Figure 3), which can display the aforementioned classes of visual signals and their placement on the $\mathrm{AV}$ can be varied in order to evaluate pedestrians' perceptions and responses to different intentions. We also aspire to examine the relationship between varying levels of abstraction in communicated information and their subjective and cognitive perception by the pedestrians.

\section{REFERENCES}

[1] Hamed S. Alavi, Farzaneh Bahrami, Himanshu Verma, and Denis Lalanne. 2017. Is Driverless Car Another Weiserian Mistake?. In DIS'17 Companion. 249-253. https://doi.org/10.1145/3064857.3079155

[2] Vicky Charisi, Azra Habibovic, Jonas Andersson, Jamy Li, and Vanessa Evers. 2017. Children's Views on Identification and Intention Communication of Self-driving Vehicles. In IDC'17. 399-404. https://doi.org/10.1145/3078072.3084300

[3] Grace Eden, Benjamin Nanchen, Randolf Ramseyer, and Florian Evéquoz. 2017. On the road with an autonomous passenger shuttle: integration in public spaces. In CHI'17 Extended Abstracts. 1569-1576.

[4] Nikhil Gowda, Wendy Ju, and Kirstin Kohler. 2014. Dashboard Design for an Autonomous Car. In AutomativeUI'14. 1-4.

[5] Nicolas Guéguen, Sébastien Meineri, and Chloé Eyssartier. 2015. A pedestrian's stare and drivers' stopping behavior: A field experiment at the pedestrian crossing. Safety science 75 (2015), 87-89.

[6] Lynn M Hulse, Hui Xie, and Edwin R Galea. 2018. Perceptions of autonomous vehicles: Relationships with road users, risk, gender and age. Safety Science 102 (2018), 1-13.

[7] Kyeil Kim, Guy Rousseau, Joel Freedman, and Jonathan Nicholson. 2015. The travel impact of autonomous vehicles in metro atlanta through activity-based modeling. In The 15th TRB National Transportation Planning Applications Conference.

[8] Jeamin Koo, Jungsuk Kwac, Wendy Ju, Martin Steinert, Larry Leifer, and Clifford Nass. 2015. Why did my car just do that? Explaining semi-autonomous driving actions to improve driver understanding, trust, and performance. International Journal on Interactive Design and Manufacturing (IJIDeM) 9, 4 (2015), 269-275.

[9] Victor Malmsten Lundgren, Azra Habibovic, Jonas Andersson, Tobias Lagström, Maria Nilsson, Anna Sirkka, Johan Fagerlönn, Rikard Fredriksson, Claes Edgren, Stas Krupenia, et al. 2017. Will There Be New Communication Needs When Introducing Automated Vehicles to the Urban Context? In Advances in Human Aspects of Transportation. Springer 485-497.

[10] Karthik Mahadevan, Sowmya Somanath, and Ehud Sharlin. 2017. Communicating Awareness and Intent in Autonomous Vehicle-Pedestrian Interaction. Technical Report. Science.

[11] Milecia Matthews, Girish Chowdhary, and Emily Kieson. 2017. Intent Communication between Autonomous Vehicles and Pedestrians. arXiv preprint arXiv:1708.07123 (2017)

[12] Alexander Meschtscherjakov, Alina Krischkowsky, Katja Neureiter, Alexander Mirnig, Axel Baumgartner, Verena Fuchsberger, and Manfred Tscheligi. 2016. Active corners: Collaborative in-car interaction design. In DIS'16. 1136-1147.

[13] Jonas Meyer, Henrik Becker, Patrick M Bösch, and Kay W Axhausen. 2017. Autonomous vehicles: The next jump in accessibilities? Research in Transportation Economics 62 (2017), 80-91.

14] Adam Millard-Ball. 2018. Pedestrians, Autonomous Vehicles, and Cities. Journal of Planning Education and Research 38, (2018), 6-12.

[15] Enrica Papa and António Ferreira. 2018. Sustainable Accessibility and the Implementation of Automated Vehicles: Identifying Critical Decisions. Urban Science 2, 1 (2018).

[16] Zeheng Ren, Xiaobei Jiang, and Wuhong Wang. 2016. Analysis of the Influence of Pedestrians' eye Contact on Drivers' Comfort Boundary During the Crossing Conflict. Procedia engineering 137 (2016), 399-406.

[17] Dirk Rothenbücher, Jamy Li, David Sirkin, Brian Mok, and Wendy Ju. 2016. Ghost driver: A field study investigating the interaction between pedestrians and driverless vehicles. In RO-MAN. 795-802. 